\title{
Hydrocephalus Revisited: New Insights into Dynamics of Neurofluids on Macro- and Microscales
}

\author{
Hans C. Ludwig ${ }^{10}$ Hans C. Bock ${ }^{10}$ Jutta Gärtner ${ }^{2(}$ Stina Schiller ${ }^{2}$ Jens Frahm ${ }^{3}$ (1) \\ Steffi Dreha-Kulaczewski ${ }^{2}$
}

${ }^{1}$ Division of Pediatric Neurosurgery, Department of Neurosurgery,
University Medical Center Göttingen, Göttingen, Germany
2 Division of Pediatric Neurology, Department of Pediatrics and
Adolescent Medicine, University Medical Center Göttingen,
Göttingen, Germany
${ }^{3}$ Biomedical NMR, Max Planck Institute for Biophysical Chemistry,
Göttingen, Germany

Address for correspondence Hans C. Ludwig, MD, Division of Pediatric Neurosurgery, Department of Neurosurgery, University Medical Center Göttingen, Robert-Koch-Str. 40, 37075 Göttingen, Germany (e-mail: hludwig@med.uni-goettingen.de).

Neuropediatrics 2021;52:233-241.

\author{
Abstract \\ Keywords \\ - cerebrospinal fluid \\ - real-time MRI \\ - hydrocephalus \\ - space flight disease \\ - aquaporin \\ - spontaneous \\ intracranial \\ hypotension \\ - neural tube defect
}

New experimental and clinical findings question the historic view of hydrocephalus and its 100-year-old classification. In particular, real-time magnetic resonance imaging (MRI) evaluation of cerebrospinal fluid (CSF) flow and detailed insights into brain water regulation on the molecular scale indicate the existence of at least three main mechanisms that determine the dynamics of neurofluids: (1) inspiration is a major driving force; (2) adequate filling of brain ventricles by balanced CSF upsurge is sensed by cilia; and (3) the perivascular glial network connects the ependymal surface to the pericapillary Virchow-Robin spaces. Hitherto, these aspects have not been considered a common physiologic framework, improving knowledge and therapy for severe disorders of normal-pressure and posthemorrhagic hydrocephalus, spontaneous intracranial hypotension, and spaceflight disease.

\section{Introduction}

\section{Challenging the Historic View}

Brain disorders arising from disturbed cerebrospinal fluid (CSF) circulation constitute a major proportion of human diseases. Incidences of 49.5/100,000 have been found for prevalent forms such as isolated hydrocephalus (HC) and as high as $81.2 / 100,000$ for HC related to prematurity. ${ }^{1}$ In fact, children represent the majority of patients affected by HC, often in conjunction with congenital neural tube defects, brain malformations, and posthemorrhagic hydrocephalus (PHHC). As a consequence, $\mathrm{HC}$ is the most frequent reason for brain surgery during childhood. Epidemiologic studies demonstrate the influence of economic status; in high-income countries, children mostly suffer from PHHC, while in low- and mediumincome countries, $\mathrm{HC}$ predominantly emerges as a sequela of neural tube defects and congenital anomalies. ${ }^{2}$ On the other

received

September 2, 2020

accepted after revision

March 7, 2021

published online

June 30, 2021

hand, reflecting the demographic shift with the increasing incidence of neurodegenerative disorders, there has been an increase in elderly patients diagnosed with idiopathic normalpressure hydrocephalus (iNPH) with a mortality rate of more than $80 \%$ over 5 years when untreated. ${ }^{3}$

Our current pathophysiologic concept of HC is largely based on the hypothesis of bulk flow and Dandy's classification of communicating and obstructive HC derived from a small number of animal experiments. ${ }^{4}$ Decades later, the hydrodynamic theory was added by Greitz, ${ }^{5,6}$ demonstrating pulsatile flow from the ventricles through the aqueduct. Klarica et al found aqueductal flow in cats to be close to zero over a period of 2 hours after open neurosurgical access to the aqueduct. ${ }^{7}$ These results contradicted earlier assumptions of unidirectional CSF routes from the choroid plexus via extracerebral cisterns to pacchionian granulations, spinal

(c) 2021. Thieme. All rights reserved. Georg Thieme Verlag KG, Rüdigerstraße 14, 70469 Stuttgart, Germany
DOI https://doi.org/ 10.1055/s-0041-1731981. ISSN $0174-304 X$. 
dural nerve roots, and cranial nerve arachnoid resorption. In later studies, ${ }^{8,9}$ the same authors concluded CSF movements to be "random" without directional preference, driven by cardiac action and respiration. Brinker et al $^{10}$ treated CSF dynamics as a diffusion process that leads to passive mixing and distribution into the different ventricular and subarachnoid compartments, while Buishas et al postulated CSF exchange by osmosis. ${ }^{11}$

Recent findings challenge these views about CSF circulation and call for a physiologic concept that takes the interplay of macroscopic fluid flow and microscopic drainage pathways into account. ${ }^{12}$ New insights particularly apply to (1) the discovery of mainly respiration-induced CSF dynamics by real-time magnetic resonance imaging (MRI), ${ }^{13,14}(2)$ novel details about the regulatory cascades of cerebral aquaporin water channels, ${ }^{15}$ and (3) increasing clinical expertise regarding disorders of perturbed CSF circulation. ${ }^{16}$ This review brings together the current thoughts on the multicompartmental dynamics of neurofluids to foster a better understanding of CSF disorders such as HC accompanying Chiari malformations, rhinoliquorrhea, and spontaneous intracranial hypotension (SIH). Moreover, aspects about cerebral fluid dynamics in zero gravity are included. The important findings about changes of brain volumes during space flights just recently been published are perfectly in line with the concept of HC discussed here. Hence, their discussion will contribute greatly to the understanding of the new point of view.

\section{Breathing, CSF Dynamics, and Venous Flow}

Noninvasive flow MRI techniques play a pivotal role in the research of human HC because they offer quantitative evaluations of both CSF movement and blood flow. ${ }^{17}$ Extending conventional techniques, real-time MRI developments now allow for dynamic assessments of the functional anatomy and fluid flow at high spatial and temporal resolution. ${ }^{13}$ Most importantly, real-time MRI studies may be performed without the need for physiologic gating. Physiologic gating to date affects almost all flow MRI studies by restricting the results to processes governed by cardiac periodicity. Without such prejudice, real-time MRI has facilitated the discovery of respiration-induced CSF transport ${ }^{14}$ and elucidated the tightly connected interplay between the venous fluid systems and the observed CSF upward surge. ${ }^{18}$ Similar results of a CSF flow through the aqueduct and into the ventricles could be shown by time-SLIP MRI techniques first presented by Yamada et al, ${ }^{19}$ although these purely qualitative measurements preclude quantitative assessments of flow direction, velocities, and volumes. In fact, CSF movement into the head during inspiration is accompanied by downward blood flow within the veins of the head/neck region, as demonstrated in spinal epidural veins (-Fig. 1). The pooling of venous blood in large extracranial collecting veins ensures cardiac preload, which then leads to adequate systolic ejection according to the Frank-Starling law.

In contrast to that of extraspinal cervical veins, which are equipped with valves, the high-capacity system of the epidural venous plexus remains valveless. ${ }^{20,21}$ Epidural veins

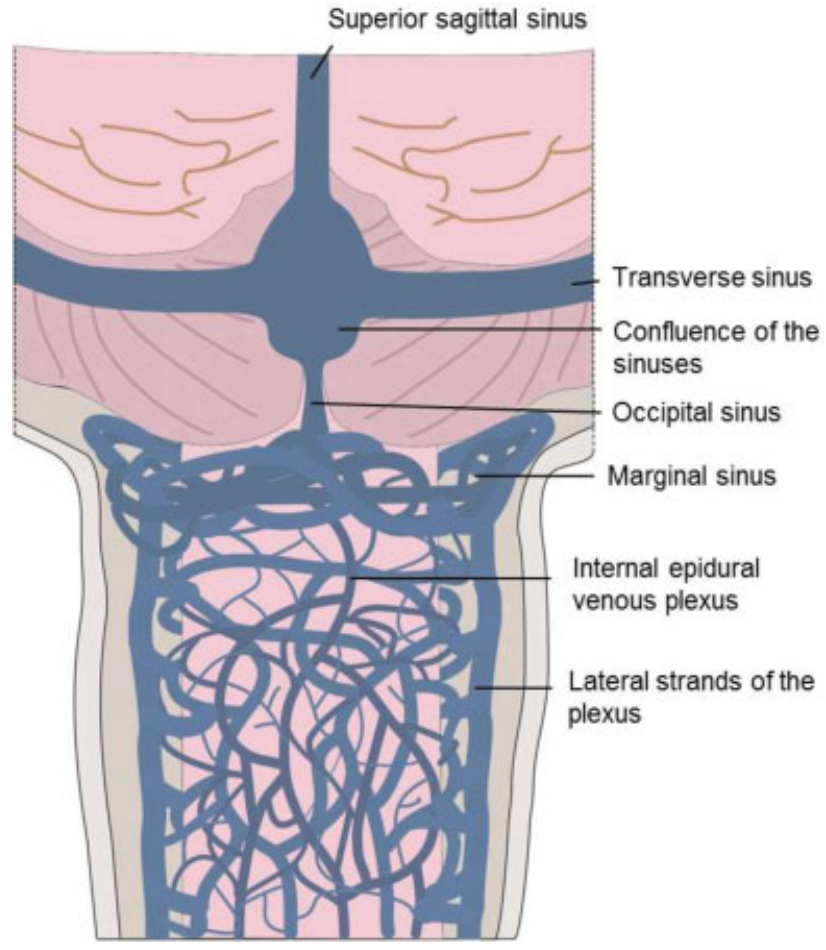

Fig. 1 Coronal view of the posterior fossa with the major dural sinuses (bilateral transverse sinus and singular sagittal sinus) and their confluence. The smaller occipital sinus connects to the marginal sinus lining the foramen magnum. From here, direct connections to the epidural venous plexus of the upper spinal canal exist. Thick strands at both sides of the spinal cord form the lateral parts of the usually rope ladder-like cervical venous plexus, which can be detected by flow MRI. Abundance of connections between intracranial and intraspinal venous compartments through the craniocervical junction are modified from figures by Henriques ${ }^{20}$ showing resin casts of vertebral veins.

are kept open by negative pleural and epidural transmural venous pressure even under mechanical ventilation. ${ }^{22}$ This well-known phenomenon can be observed during lumbar puncture when the pendant saline drop at the needle opening gets aspirated as soon as the tip reaches and penetrates into the epidural space regardless of posture. ${ }^{23}$ This observation is valuable for excluding any suspected bias from CSF dynamic studies performed in the supine position. The epidural venous plexus accompanies the dural sac throughout its entire length. It forms manifold connections between intra- and extraspinal spaces and thoracic and abdominal cavities. Dreha-Kulaczewski et al ${ }^{18,24}$ demonstrated the enhanced filling of spinal epidural veins during inspiration followed by draining during expiration. The respiratory-induced flow changes in this important venous drainage pathway can be transmitted into the filling and emptying of the directly connected venous compartments of the posterior fossa (-Fig. 1). Following the Monro and Kellie doctrine of constant intracranial volume, the volume of fluid leaving the cranial vault must be counterbalanced. Thus, the upward movement of CSF and the associated venous outflow compensate for each other during respiration.There is longstanding discussion in the literature regarding the dynamic of net CSF flow, whether by cardiac pulsatility or fluid 
displacement by respiration and each individual contribution. To separate cardiac- and respiratory-driven CSF motion, Takizawa et al performed asynchronous 2D phase-contrast MRI experiments under respiratory guidance. They found that for velocity, the cardiac component was greater than the respiratory component. In contrast, for the amount of volume displacement, the respiratory component was greater. ${ }^{25}$ This pattern of fast and small cardiac-driven modulations but slow and high displacement by respiration was confirmed by the study of Yatsushiro et al. ${ }^{26}$ Yildiz et al were able to demonstrate a comparable contribution of respiration and cardiac pulsations on CSF dynamics through the aqueduct during deep breathing but not during natural breathing. ${ }^{27}$ This pattern could resemble the situation during deep nonrapid eye movement (NREM) sleep. ${ }^{28}$

From this perspective, the posterior fossa of the skull might be considered a transfer unit. Further upward, the aqueductal tube connecting the fourth and third ventricles constitutes a singular gate into the supratentorial brain CSF spaces. It might even be hypothesized that it serves as a control valve for the passage of large flow volumes during inspiration. Given that respiration-related CSF flow is continuously subjected to more rapid and low-amplitude modulating fluctuations caused by cardiac pulsatility, ${ }^{25}$ the thin diameter of the aqueduct results in laminar flow and enables instantaneous control of CSF flow volumes according to the law of Hagen-Poiseuille. ${ }^{16}$ It accounts for the radial diameter of the aqueduct to the fourth power, which renders the structure predestined as a fine-tuned system for CSF dynamic flow into and out of the brain ventricles. ${ }^{29}$ This hypothesis is supported by the anatomic details of the aqueductal environment, in which the frontal prepontine Liliequist membrane and the dense arachnoid membranes of the dorsal cisterna quadrigemina ${ }^{30}$ prevent any bypassing subarachnoid para-aqueductal exchange of CSF, which would jeopardize the tuning capabilities (-Fig. 2). Any flow of CSF through the cisterns bypassing the aqueduct, as reported by studies based on experiments in rats, ${ }^{31}$ would prevent aqueductal stenosis and obstructive $\mathrm{HC}$ in humans. The cisterna quadrigemina itself, as a protrusion of the third ventricle, might be able to promote and transmit intraventricular pressure immediately to the aqueduct, thereby influencing the tube diameter (-Fig. 2). ${ }^{32}$

A very important aspect of the tightly connected and wellbalanced CSF and venous systems is to ensure upward movements of CSF against hydrostatic pressure. This physiologic principle is a prerequisite for a sustained upright body position because it maintains brain turgor and thus adequate intracranial CSF volume. Above all, hydrostatic uplift or buoyant forces inside the ventricles and arachnoidal spaces must be ensured in all body positions as well as over long periods of significant skull growth, as brain volume increases from infancy until adolescence. In view of the physiologic consequences of an upright position, findings from quadrupeds may therefore be misleading, as their aqueducts are oriented differently with respect to gravity and hydrostatic pressure. Further differences might be due to the much smaller dimensions of intracranial fluid spaces and absorption structures. Sheep, for example, are able to absorb up to $50 \%$ of their CSF volume by cervical vessel structures. ${ }^{33}$ It should also be noted that the majority of experimental studies have been conducted in very young animals in which CSF structures and dynamics are not yet fully developed-comparable with infants without pacchionian granulations during the first year of life. ${ }^{34}$ The exact volumes of reabsorption in adults and children above 4 years of age are still under debate. The CSF circulates at a daily rate of around $500 \mathrm{~mL}$. The concepts of the reabsorption process by perivascular aquaporin channels and Virchow-Robin spaces (VRS) have to be separated from respiration- and cardiac pulsatility-induced

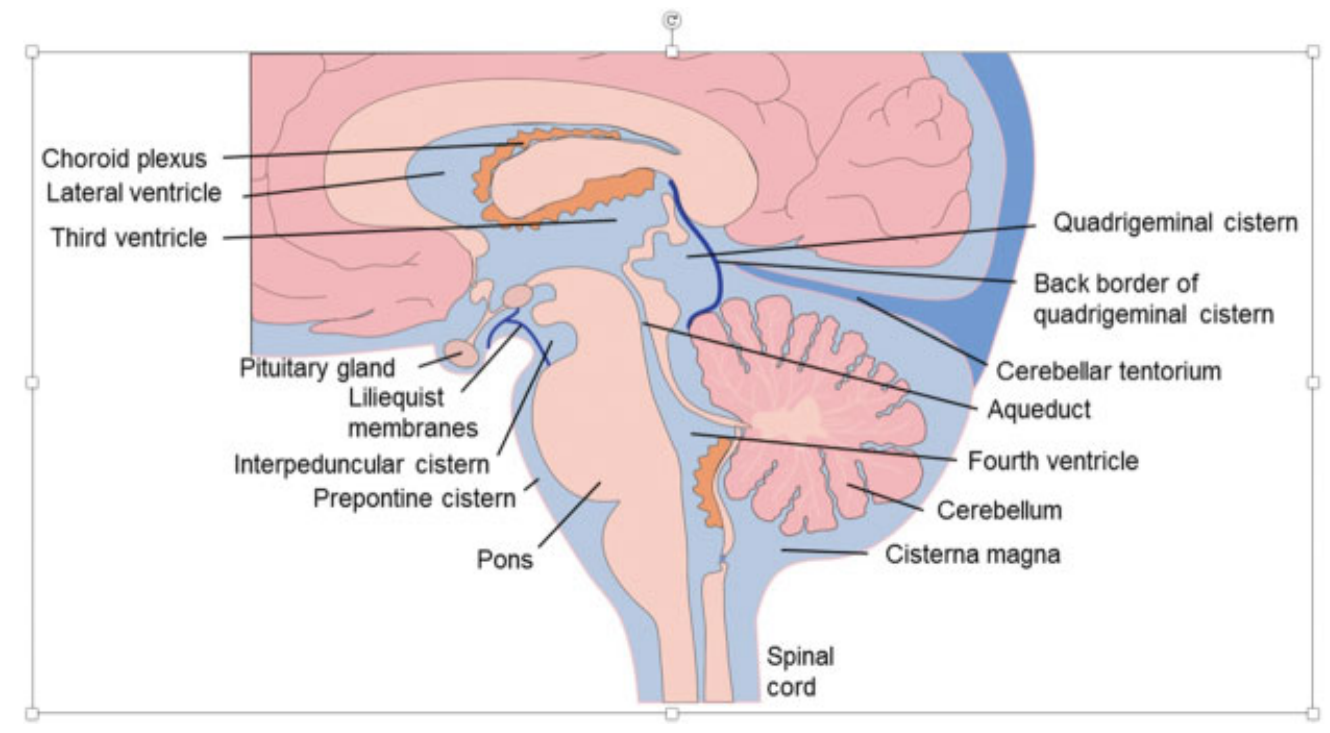

Fig. 2 Sagittal view of the internal CSF spaces and main cisterns constituting external CSF spaces. The Liliequist membranes (dark blue) block the prepontine pathways of CSF (modified from Hussein et $\mathrm{al}^{32}$ ). The line (dark blue) outlining the quadrigeminal cistern represents the complex webs of dense arachnoid membranes hampering CSF flux. Note the unique anatomical position of this cistern. Here, variations in size or pressure may foster the modulation of aqueductal diameter, thereby regulating CSF flow into or out of brain ventricles. 
dynamics. Reabsorption might be dependent more on the glymphatic system and its molecular features.

\section{Ependymal Fluid Flow}

Ependymal cells cover the outer surface of the choroid plexus and the inner wall of the brain ventricular system as well as the central canal of the spinal cord. These unique structures can be damaged by infections, tumors, and subarachnoid hemorrhage (SAH) or intraventricular hemorrhage (IVH) due to germinal matrix disruption in prematurity. The latter is described as a bleeding-induced N-cadherin-mediated disruption of the ependymal junctions and is usually followed by severe and catastrophic destruction of the ependymal lining of the ventricular wall. ${ }^{35}$ The ventricular wall encloses, protects, and organizes one of the most delicate structures of the central nervous system, the subventricular zone, also called "the lake front property." ${ }^{36}$ It harbors valuable stem cells essential for repair and plasticity until adulthood. The disturbance of this zone arising from, e.g., germinal matrix bleeding from PHHC, prematurity, chronic hypoxia, and inflammation is one of the major causes of the lifelong disability associated with periventricular leucomalacia. ${ }^{37}$

The cilia of these ependymal cells provide a flow-sensing system that represents a regulatory mechanism serving a variety of purposes. In addition to promoting active CSF transport near the ventricular walls, these cilia are capable of detecting flow directions, ${ }^{38}$ turbulence patterns, and disturbances in circadian rhythms. ${ }^{39}$ For example, ventricular dilation without an increase in intracranial pressure (ICP) but with perturbation of CSF circulation, as accomplished by an inflatable balloon in lambs, supports the notion of ependymal fluid flow and pressure-sensing mechanisms as being responsible for the observed alterations of ventricular dimensions. ${ }^{16,40}$ Disturbances of the sensing cilia system have been identified as being responsible for alterations in ventricular morphology and dimension. ${ }^{41}$ The ability of the ependyma to sense CSF flow properties in conjunction with the aqueduct provides a means to fine-tune and adapt CSF circulation for acute physiologic demands. ${ }^{42}$

Notably, pediatric HC can be evoked not only by CSFrelated pathologies but also by underlying structural anomalies such as schizencephaly, aplasia, or severe cortical dysplasia and migration deficits.

\section{Bergmann Glia and Astrocytic Fluid Flow}

A crucial process of the ventricular system during embryonic development is the formation of a CSF-filled tube. This structure consists of neuroepithelial cells arranged in tubular forms conducting fluid and precursor CSF and providing steady turgor with a continuous flow of signaling peptides to control progenitor cells building up the Bergmann glia layer. ${ }^{43-45}$ Bergmann glial cells are characterized by a unique polarity extending between the ventricular wall and the glia limitans, and they mature later to astrocytic layers. The glia limitans consists of extracellular matrix laminin and fibronectin ${ }^{46-48}$ contacting and surrounding the capillary wall ${ }^{47}$ as well as the basal membrane of the ependyma. The astrocytic layer is essential for the brain water balancing system because the polarized astrocytic basal membrane is equipped with aquaporin-4 (AQP4), a water channel expressed in the astrocyte foot processes and ependymal cells lining the ventricles. AQP4 facilitates the transport of excess water out of the brain. ${ }^{49}$

The correct polarization of Bergman glia and later astrocytes between the capillary wall and subependymal glia is mandatory for water homeostasis in the brain. Disturbances of this system by hemoglobin ${ }^{50}$ in preterm IVH lead to deleterious lesions by destroying the $\mathrm{N}$-cadherin-dependent cell junctions. ${ }^{35}$ This alteration is responsible for the regional destruction of the ependymal cell layer and activation of extracellular matrix proteins around the astrocyte membrane as well as for the reported loss of polarized AQP4. ${ }^{51}$ The condition is not restricted to prematurity and could also be identified in congenital and even adult $\mathrm{HC}^{52,53}$

AQP4 is responsible for the recycling of brain water and cellular waste by connecting intercellular and glymphatic flow with CSF circulation. The system prevents cardiac- and respiration-related pulsatility from propagating to the interstitial fluid compartment of the brain. Exposure to those dominant forces jeopardizes the blood-brain barrier and precludes any autoregulative vasomotor control of capillary flow by the direct transduction of systemic blood pressure. ${ }^{48}$ However, without an alternative hydrodynamic mechanism, the brain interstitial fluid remains stagnant. As noted by Nakada, ${ }^{54}$ the AQP4-supported influx of water into the pericapillary VRS generates continuous interstitial flow, which constitutes an essential part of the clearance system for $A \beta$-amyloid. Both adequate ventricular CSF circulation and interstitial flow in VRS are mandatory for proper neurofluid circulation (-Fig. 3 ). These interacting systems control the brain water balance under diverse conditions ranging from growth to standing and prone positions under variable systemic blood pressures as well as during physical exercise and sleep. Taken together, the combined understanding of functional neurofluids at the macroscopic, microscopic, and molecular levels may be exploited to re-evaluate the pathophysiology of conditions with disturbed CSF distribution and transport. At this point in time, aspects discussed here are still conceptual, derived from the novel in vivo insights facilitated by real-time flow MRI and supported by the literature.

\section{CSF Disorders and Beyond}

\section{Chiari Malformations}

The neural tube of the fetus usually closes around embryonic day 25. Incomplete closure results in open spina bifida or fetal meningomyelocele (MMC), which forms a CSF fistula over the remaining months of pregnancy until surgical closure by either fetal intrauterine or postnatal surgery. A study of the effectiveness of intrauterine MMC repair demonstrated that intrauterine closure at approximately the 24th week of pregnancy significantly reduced the rate of Chiari II malformation with less tonsillar herniation and HC. ${ }^{55}$ The lack of distention of the embryonic posterior fossa had already been postulated as the underlying mechanism decades ago. ${ }^{56}$ After surgical closure of the MMC fistula, 


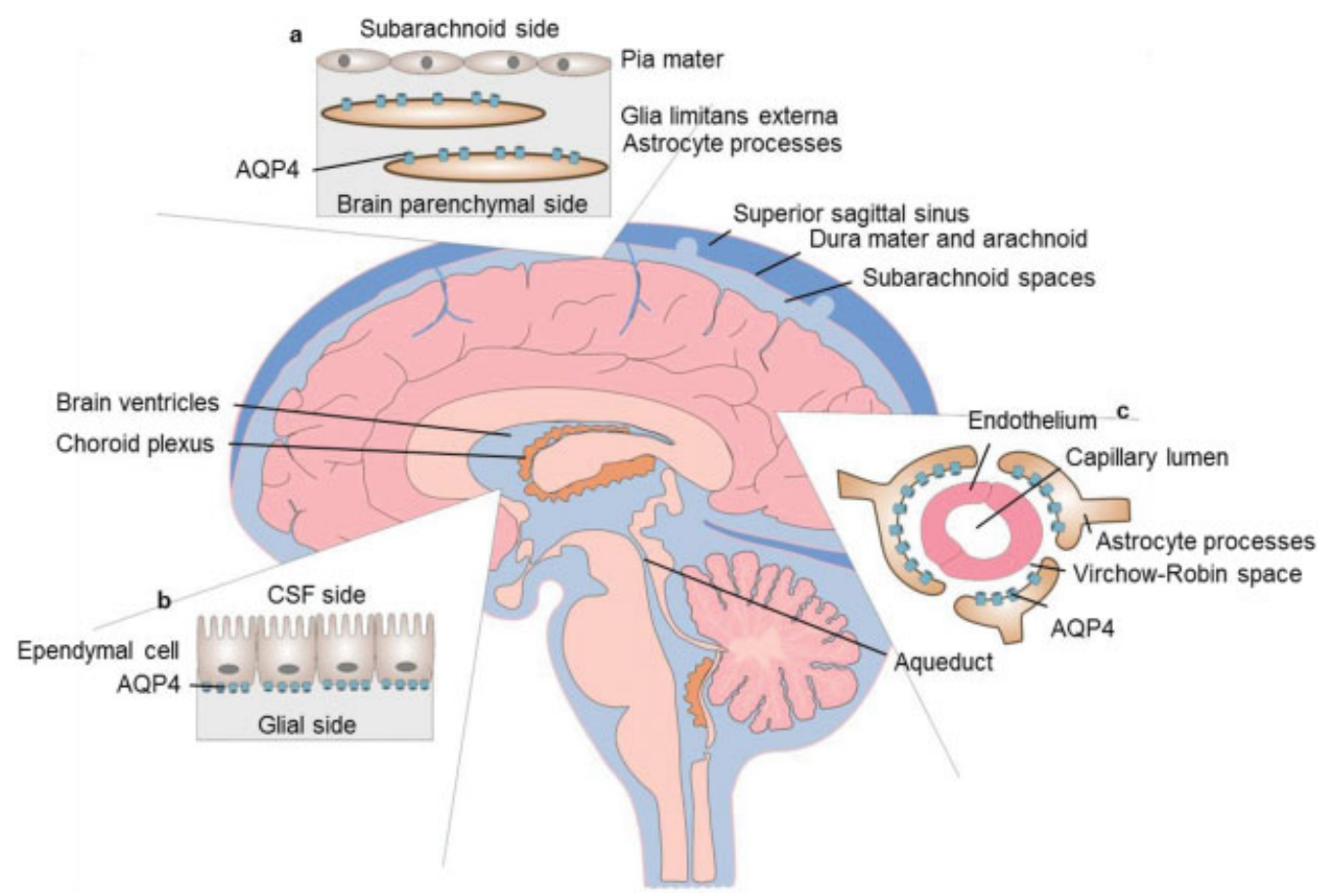

Fig. 3 Sagittal view of the brain and CSF spaces. Insets illustrate the main location of aquaporin-4 water channel (APQ4) expression (modified from Nakada et $\mathrm{a}^{49}{ }^{4}$. Astrocytes harboring the AQP4 system in their end-feet processes are essential composites of brain tissue-CSF and brain tissue-blood interfaces. (a) Astrocytes of the glia limitans externa (situated subpial) expressing AQP4 are part of the outer brain barrier between the subdural spaces and outer brain surface. (b) AQP4 is expressed on the glial/brain parenchymal side of the ependymal cell layer, which covers all internal CSF spaces. (c) Astrocyte end feet enclosing pericapillary spaces referred to as Virchow-Robin spaces (VRS) are equipped with AQP4 water channels and contribute to the blood-brain barrier. Here, the astrocytic AQP4 system regulates water influx into the VRS and generates interstitial flow.

sufficient upward movement of CSF ensures appropriate ventricular filling and growth of the posterior fossa. Remarkably, at approximately the 24th gestational week, fetal respiration emerges. ${ }^{57}$ Assuming the same physiologic principles as those postnatally, the inspiratory extension of the thoracic cavity and lowering of the intrathoracic pressure probably induces venous outflow from intracranial venous compartments toward the heart, as the most important factor for cranial fluid flow. ${ }^{57}$ Breathing movements of the thorax govern the transport of amniotic fluid instead of air in and out of the lungs. The venous fluid shifting out of the cranium has thus to be counterbalanced by upwardly directed CSF flow.

The establishment of adequate prenatal CSF dynamics is a prerequisite not only for the development of brain structures and skull size but also for neuronal migratory processes. Fetuses with spina bifida aperta without prenatal surgical treatment present significantly impaired neuronal migration of the subventricular zone with consecutive heterotopia, damage of the ependymal layers with regional loss of the ependyma, desynchronized ciliary beating, and resultant astrocytic scarring. ${ }^{58,59}$ All of these factors are also known to promote the formation of $\mathrm{HC}$.

\section{Spontaneous Intracranial Hypotension and Rhinoliquorrhea}

$\mathrm{SIH}$ is a severe pathophysiologic condition affecting adults up to the age of 60 years, but it very rarely affects children. Commonly, osseous spurs inside the spinal canal cause dural leakage and epidural collection of CSF inside the canal below the cerebellar tentorium. Depending on the loss of CSF, cranial hypotension may ensue, leading to subdural effusion and chronic subdural bleeding. Subsequently, characteristic signs of brain sagging, such as a lowered cerebellum and tonsillar herniation, point out the reduced brain turgor and impaired buoyant force. The condition, termed nongeriatric chronic subdural hematoma, can cause severe morbidity and even mortality. Therapy includes the neurosurgical application of spinal blood patches, bed rest, and, if possible, closure of the leakage by an intraspinal intradural approach. ${ }^{60,61}$

In contrast to infratentorial leakage, CSF loss due to traumatic rhinoliquorrhea, for example, at the petrous bone or in frontobasal locations, occurs within the supratentorial subarachnoid CSF spaces. It is important to note that although severely complicated by local infections or meningitis, brain sagging alluding to SIH with loss of buoyancy has not been observed. Symptoms such as severe headache and vomiting exaggerated in an upright position are similar to those after lumbar puncture and consecutive temporary shortage of CSF. Therapeutic measures with aminophylline and caffeine have been proven effective. ${ }^{62}$ Notably, both substances elevate breathing frequency, which, considering it the main driving force, could result in more profound CSF being moved upward into the cranial vault.

The important difference between loss of CSF in rhinoliquorrhea permanent spinal leakage is the relative location to the aqueduct. The capacity to counterbalance continuous fluid loss below the aqueduct by breathing-induced upward movements of CSF is limited by the shortage of available CSF 
volume; thus, SIH and chronic subdural hematoma may evolve over time. On the other hand, CSF loss due to subarachnoid leakage above the aqueduct obviously does not induce SIH and has not been reported. Extending our hypothesis about the potential valve function of the aqueduct in conjunction with dense sealing of the para-aqueductal pathways (-Fig. 2), the exclusive fluid passage seems to be able to prevent intracerebral hypotension.

\section{Shunt-Mediated CSF Overdrainage}

An upright body position and concomitant hydrostatic forces represent a challenge for the treatment of CSF overdrainage using ventricular shunt systems. CSF overdrainage into the extracranial compartments is known to be a common side effect eventually associated with severe and acutely exacerbating complications, particularly during periods of fast growth in childhood and adolescence. Slit ventricles detected by MRI and complaints of severe headaches that escalate during movement into a standing position are typical symptoms. Their increase over the course of the day might be attributed to a diminution in systemic hydration and the consequently increasing imbalance of the venous-CSF fluid system. Affected children usually develop learning difficulties and complain about headaches and dizziness when moving from a horizontal position.

It is compelling to note that fluid overdrainage from the ventricles apparently does not provoke the characteristic sequela of $\mathrm{SIH}$, as brain sagging has never been observed. Moreover, headaches early in the morning are comparatively rare, which may be attributed to the night-long filling effect due to breathing-related upward CSF movement from the spinal canal into the ventricles, i.e., during sleep in a horizontal position and under hydrostatic equilibrium. Over time, however, ventricular narrowing occurs and may lead to the encasement of shunt catheters between the walls. Chronic overdrainage may even cause subdural effusion and bleeding. The treatment of this condition requires the integration of a gravity control system into the shunt diversion to attenuate hydrostatic forces.

\section{Space Flight Disease}

Recently, Van Ombergen et $\mathrm{al}^{63,64}$ reported persistent enlargement of the brain CSF spaces in astronauts after longduration space flights. Postflight volume changes in the lateral ventricle were shown to be positively correlated with visual acuity loss-a well-known sequela of space flight-associated neuro-ocular syndrome (SANS). The underlying mechanisms of SANS, which refer to various ocular abnormalities such as globe flattening and optic disc edema, are still not fully understood. The current findings demonstrate not only long-lasting enlargement of ventricular and subarachnoid CSF spaces but also postflight reductions in white and gray matter volumes. This observation seems to link changes in brain CSF spaces to the formation of SANS. In fact, the observations of increased ventricular volume and decreased CSF space below the vertex highly support disturbances of CSF circulation as the underlying cause, possibly due to imbalances of breathing-induced CSF upward flow, as hypothesized by Ludwig et al. ${ }^{65}$ Interestingly, these alterations are sustained for as long as 7 months after space flight, implying that they eventually remain unaffected for a longer time by the return to gravity and the reestablishment of hydrostatic pressure conditions. This might indicate that after gravity is restored, the human system needs quite a long time for the excessive accumulation of CSF in the brain ventricles as well as the white and gray matter volumes to normalize and for the CSF flow system to reach physiological conditions.

Thus, long-term changes in ventricular volumes due to space flight might act as a model for the induction of HC.

Notably, the brain aquaporin system is able to adapt to different pressure situations ${ }^{66,67}$ by adjusting the localization of aquaporin-1 (AQP1), an isoform of aquaporin water channels expressed at the ependyma of the choroid plexus, ${ }^{68}$ and the expression density of AQP4 at astrocytic end feet. In mice experiencing space flights or head-down tilt (HDT), AQP1 was redistributed from apical to basal ependymal membranes. ${ }^{69}$ AQP expression was altered by gravity. ${ }^{70}$ In mice with kaolininduced HC, AQP1 was redistributed from the apical to cytoplasmic vesicles and basal membranes of the ependyma. ${ }^{71}$ Hence, alterations in the aquaporin system might be responsible for long-lasting or permanent adaptations of ventricular volumes after zero-gravity space flights and might be involved in many CSF disorders in which CSF dynamics are altered. A related pathway has been described for nitric oxide (NO), ${ }^{72}$ which is delivered by neuronal or endothelial isozymes and is able to cross membranes ${ }^{73,74}$ and AQP4 channels. It facilitates the motility and beating frequency of several ciliated epithelial cells. ${ }^{75}$ A disturbance of the NO system by intraventricular hemoglobin in preterm infants or $\mathrm{SAH}^{76}$ allows us to argue for a link between CSF flow, ependymal ciliary sensing function, endothelial relaxation, capillary dilation, and astrocytic clearance function. NO is also important for the structural maintenance of the periventricular subependymal zone. ${ }^{77}$ It may therefore be worthwhile to focus on elevated NO expression in microgravity as well as in gravity simulation experiments by rat tail suspension or during human HDT. ${ }^{78}$

\section{Sleep and Head-down Tilt}

Based on the observation that inspiration elicits an upward cranial motion of CSF, it can be assumed that sleep in a horizontal body position with zero hydrostatic pressure enhances fluid flow, while irregular breathing during sleep may lead to CSF-related disorders. For example, obstructive sleep apnea has been reported to be frequently associated with the development of iNPH. ${ }^{79}$ This may be due to a disturbance in the well-orchestrated interplay between the venous and CSF systems. During sleep apnea, repeated attempts to breath induce strong respiratory efforts against a closed glottis, which severely lowers the intrathoracic pressure. The subsequently compromised venous return to the heart may result in retrograde intracranial venous hypertension. Perturbations of venous-CSF equilibrium hamper CSF exchange between the spinal canal and head and may thus contribute to an increasing ventricular diameter and reduced glymphatic circulation. ${ }^{80,81}$ 
Enhanced upward CSF flow by inspiration during sleep was observed in NREM sleep in healthy individuals using electrophysiologic methods and blood oxygenation-level dependent MRI. ${ }^{28}$ During NREM phases of deep sleep, ${ }^{82}$ the human respiration cycle is presumably slow, regular, deep, and sustainable enough to move a sufficient CSF volume into the ventricles, probably facilitated by a reduction in the hydrostatic gradient. Such an ample supply of CSF might be the reason for the enhanced glymphatic clearance of tau and $A \beta$-amyloid during sleep. ${ }^{83,84}$

An HDT position reverses hydrostatic conditions and leads to higher ICP and may serve as an experimental simulation of long-duration space flight with zero gravity. Recent studies have reported decreased cerebral blood flow in patients in an HDT position compared with a horizontal position. Furthermore, exposure to $3 \% \mathrm{CO}_{2}$ during HDT enhances aqueductal CSF velocities and lateral ventricle CSF mean diffusivity. ${ }^{85,86}$ Remarkably, a simultaneous increase in lateral ventricle volume and the formation of optic disc edema in the HDT position resemble observations in astronauts after space flight. ${ }^{70,85,87}$ In accordance with animal models of $\mathrm{HC},{ }^{53}$ altered ICP, fluid flow physiology, and dynamics during HDT may be hypothesized to interfere with choroidal depolarization and AQP dysfunction. ${ }^{88}$

\section{Discussion}

The recent observation of breathing-induced CSF upward motion in association with intrathoracic venous pooling opens new horizons for a profound understanding of CSF flowrelated disorders. These insights apply to CSF homeostasis in pediatric diseases such as preterm PHHC and congenital obstructive $\mathrm{HC}$ as well as in adult intracranial spontaneous hypertension. Additional information may be gathered by observations of altered CSF physiology during space flight. Zero gravity offers a model to more precisely understand conditions that are related to the counterbalancing effects of gravity, venous outflow, and CSF upward flow. On the other hand, CSF exchange with interstitial fluid and VRS relies on proper expression of AQP4 water channels in astrocytic end feet. Thus, CSF regulation comprises at least three main regulatory mechanisms: (1) inspiration-induced CSF supply upward into the skull and control of CSF flow into brain CSF spaces by aqueductal function, (2) ependymal flow and its pressure-sensing properties, and (3) AQP1 and AQP4 functions regulating interstitial water secretion and resorption on a molecular scale, respectively. Such knowledge is mandatory to explain iNPH, PHHC, SIH, and spina bifida-related disorders as well as space flight disease. Furthermore, this knowledge may help us to further understand conditions associated with CSF disturbances and, above all, to develop and improve therapeutic approaches.

\section{Search Strategy and Selection Criteria}

We searched PubMed, Crossref Search, and Web of Science for reports published in English from January 1, 1995, to March 31, 2020. The search terms "hydrocephalus" and "hydrocephalic" were combined with many terms such as "epidemiology," “pathophysiology," “etiology," “diagnosis," "management," "aquaporin," "cerebro spinal fluid," and "glymphatic." In addition to the search results, we also hand searched the references of relevant articles retrieved by search strategy. The final reference list was generated on the basis of relevance to the topics covered in this review.

\section{Author Contributions}

H. C. L., S. D.-K., and J. F. edited the manuscript; H. C. L., S. D.-K., H. C. B., and J. G. defined the scope, objective, and clinical details. H. C. L., S. D.-K., H. C. B., J. F., J.-G., and S. S. reviewed the manuscript.

\section{Competing Interests}

The authors declare no competing financial interests.

\section{Funding}

S. D.-K., H. C. L., H. C. B., and J. G. gratefully acknowledge financial support from Mrs. L. Grun funds.

\section{Conflict of Interest}

J. D. reports personal fees from Bayer, personal fees from Biogen, grants and personal fees from Novartis, and personal fees from Sanofi, outside the submitted work. In addition, J. D. has a patent "Treatment of Multiple Sulfatase Deficiency," US Patent No 16591051 licensed.

\section{Acknowledgments}

The authors gratefully thank the editors of Springer Nature Author Service for editing for proper English, style, spelling, grammar, and punctuations.

\section{References}

1 Isaacs AM, Riva-Cambrin J, Yavin D, et al. Age-specific global epidemiology of hydrocephalus: systematic review, metanalysis and global birth surveillance. PLoS One 2018;13(10):e0204926

2 Dewan MC, Rattani A, Mekary R, et al. Global hydrocephalus epidemiology and incidence: systematic review and meta-analysis. J Neurosurg 2018;37(02):1-15

3 Jaraj D, Wikkelsø C, Rabiei K, et al. Mortality and risk of dementia in normal-pressure hydrocephalus: a population study. Alzheimers Dement 2017;13(08):850-857

4 Dandy WE. Experimental hydrocephalus. Ann Surg 1919;70(02): 129-142

5 Greitz D, Wirestam R, Franck A, Nordell B, Thomsen C, Ståhlberg F. Pulsatile brain movement and associated hydrodynamics studied by magnetic resonance phase imaging. The Monro-Kellie doctrine revisited. Neuroradiology 1992;34(05):370-380

6 Greitz D. The hydrodynamic hypothesis versus the bulk flow hypothesis. Neurosurg Rev 2004;27(04):299-300

7 Klarica M, Oresković D, Bozić B, Vukić M, Butković V, Bulat M. New experimental model of acute aqueductal blockage in cats: effects on cerebrospinal fluid pressure and the size of brain ventricles. Neuroscience 2009;158(04):1397-1405

8 Klarica M, Miše B, Vladić A, Radoš M, Orešković D “Compensated hyperosmolarity" of cerebrospinal fluid and the development of hydrocephalus. Neuroscience 2013;248:278-289

9 Orešković D, Klarica M. A new look at cerebrospinal fluid movement. Fluids Barriers CNS 2014;11(01):. Doi: 10.1186/2045-8118$11-16$

10 Brinker T, Stopa E, Morrison J, Klinge P. A new look at cerebrospinal fluid circulation. Fluids Barriers CNS 2014;11(01):10-16 
11 Buishas J, Gould IG, Linninger AA. A computational model of cerebrospinal fluid production and reabsorption driven by Starling forces. Croat Med J 2014;55(05):481-497

12 Jones HC, Keep RF, Drewes LR. CNS fluid and solute movement: physiology, modelling and imaging. Fluids Barriers CNS 2020; 17:12

13 Frahm J, Voit D, Uecker M. Real-time magnetic resonance imaging: radial gradient-echo sequences with nonlinear inverse reconstruction. Invest Radiol 2019;54(12):757-766

14 Dreha-Kulaczewski S, Konopka M, Joseph AA, et al. Respiration and the watershed of spinal CSF flow in humans. Sci Rep 2018;8 (01):5594-5597

15 Papadopoulos MC, Verkman AS. Aquaporin water channels in the nervous system. Nat Rev Neurosci 2013;14(04):265-277

16 Bock HC, Dreha-Kulaczewski SF, Alaid A, Gärtner J, Ludwig HC. Upward movement of cerebrospinal fluid in obstructive hydrocephalus-revision of an old concept. Childs Nerv Syst 2019;35 (05):833-841

17 Matsumae M, Kuroda K, Yatsushiro S, et al. Changing the currently held concept of cerebrospinal fluid dynamics based on shared findings of cerebrospinal fluid motion in the cranial cavity using various types of magnetic resonance imaging techniques. Neurol Med Chir (Tokyo) 2019;59(04):133-146

18 Dreha-Kulaczewski S, Joseph AA, Merboldt KD, Ludwig HC, Gärtner J, Frahm J. Identification of the upward movement of human CSF in vivo and its relation to the brain venous system. J Neurosci 2017;37(09):2395-2402

19 Yamada S, Miyazaki M, Kanazawa H, et al. Visualization of cerebrospinal fluid movement with spin labeling at MR imaging: preliminary results in normal and pathophysiologic conditions. Radiology 2008;249(02):644-652

20 Henriques CQ. The veins of the vertebral column and their role in the spread of cancer. Ann R Coll Surg Engl 1962;31(01):1-22

21 Groen RJM, Grobbelaar M, Muller CJF, et al. Morphology of the human internal vertebral venous plexus: a cadaver study after latex injection in the 21-25-week fetus. Clin Anat 2005;18(06): 397-403

22 Nystrom EU, Blomberg SG, Buffington CW. Transmural pressure of epidural veins in the thoracic and lumbar spine of pigs. Anesthesiology 1998;89(02):449-455

23 Todorov L, VadeBoncouer T. Etiology and use of the "hanging drop" technique: a review. Pain Res Treat 2014;2014(04):1-10

24 Dreha-Kulaczewski S, Joseph AA, Merboldt K-D, Ludwig H-C, Gärtner J, Frahm J. Inspiration is the major regulator of human CSF flow. J Neurosci 2015;35(06):2485-2491

25 Takizawa K, Matsumae M, Sunohara S, Yatsushiro S, Kuroda K. Characterization of cardiac- and respiratory-driven cerebrospinal fluid motion based on asynchronous phase-contrast magnetic resonance imaging in volunteers. Fluids Barriers CNS 2017;14 (01):25

26 Yatsushiro S, Sunohara S, Atsumi H, Matsumae M, Kuroda K. Visualization and characterization of cerebrospinal fluid motion based on magnetic resonance imaging. In: Gürer B, ed. Hydrocephalus - Water on the Brain. InTech; 2018:1-18

27 Yildiz S, Thyagaraj S, Jin N, et al. Quantifying the influence of respiration and cardiac pulsations on cerebrospinal fluid dynamics using real-time phase-contrast MRI. J Magn Reson Imaging 2017;46(02):431-439

28 Fultz NE, Bonmassar G, Setsompop K, et al. Coupled electrophysiological, hemodynamic, and cerebrospinal fluid oscillations in human sleep. Science 2019;366(6465):628-631

29 von Mecklenburg C, Håkansson CH, Lindgren M. Effects of irradiation on the cilia of the Sylvian aqueduct. A scanning electron microscopic investigation. Acta Radiol Ther Phys Biol 1974;13 (03):232-240

30 Qi S-T, Fan J, Zhang X-A, Pan J. Reinvestigation of the ambient cistern and its related arachnoid membranes: an anatomical study. J Neurosurg 2011;115(01):171-178
31 Park J-H, Park YS, Suk J-S, et al. Cerebrospinal fluid pathways from cisterns to ventricles in N-butyl cyanoacrylate-induced hydrocephalic rats. J Neurosurg Pediatr 2011;8(06):640-646

32 Hussein S, Woischneck D, Niemeyer U. Microsurgical anatomy of the cisterna quadrigemina and cisterna velum interpositum. In: Bauer BL, Brock M, Klinger M, eds. Cerebellar Infarct. Midline Tumors. Minimally Invasive Endoscopic Neurosurgery (MIEN) Advances in Neurosurgery. Vol. 22;Berlin, Heidelberg, New York: Springer; 1994:297-302

33 Boulton M, Flessner M, Armstrong D, Hay J, Johnston M. Determination of volumetric cerebrospinal fluid absorption into extracranial lymphatics in sheep. Am J Physiol 1998;274(01):R88-R96

34 Oi S, Di Rocco C. Proposal of "evolution theory in cerebrospinal fluid dynamics" and minor pathway hydrocephalus in developing immature brain. Childs Nerv Syst 2006;22(07):662-669

35 Castaneyra-Ruiz L, Morales DM, McAllister JP, et al. Blood exposure causes ventricular zone disruption and glial activation in vitro. J Neuropathol Exp Neurol 2018;77(09):803-813

36 Ihrie RA, Alvarez-Buylla A. Lake-front property: a unique germinal niche by the lateral ventricles of the adult brain. Neuron 2011; 70(04):674-686

37 Oliver C, González CA, Alvial G, Flores CA, Rodríguez EM, Bátiz LF. Disruption of $\mathrm{CDH} 2 / \mathrm{N}$-cadherin-based adherens junctions leads to apoptosis of ependymal cells and denudation of brain ventricular walls. J Neuropathol Exp Neurol 2013;72(09):846-860

38 Kusne Y, Duran-Moreno M, Cabrales E, et al. Bi- and uniciliated ependymal cells define continuous floor-plate-derived tanycytic territories. Nat Commun 2017;8:13759

39 Faubel R, Westendorf C, Bodenschatz E, Eichele G. Cilia-based flow network in the brain ventricles. Science 2016;353(6295):176-178

40 Di Rocco C, Pettorossi VE, Caldarelli M, Mancinelli R, Velardi F. Communicating hydrocephalus induced by mechanically increased amplitude of the intraventricular cerebrospinal fluid pressure: experimental studies. Exp Neurol 1978;59(01):40-52

41 Foerster P, Daclin M, Asm S, et al. mTORC1 signaling and primary cilia are required for brain ventricle morphogenesis. Development 2017;144(02):201-210

42 Swiderski RE, Agassandian K, Ross JL, Bugge K, Cassell MD, Yeaman C. Structural defects in cilia of the choroid plexus, subfornical organ and ventricular ependyma are associated with ventriculomegaly. Fluids Barriers CNS 2012;9(01):22-35

43 Gato A, Desmond ME. Why the embryo still matters: CSF and the neuroepithelium as interdependent regulators of embryonic brain growth, morphogenesis and histiogenesis. Dev Biol 2009; 327(02):263-272

44 Korzh V. Development of brain ventricular system. Cell Mol Life Sci 2018;75(03):375-383

45 Gato A, Alonso MI, Martín C, et al. Embryonic cerebrospinal fluid in brain development: neural progenitor control. Croat Med J 2014;55(04):299-305

46 Guadagno E, Moukhles H. Laminin-induced aggregation of the inwardly rectifying potassium channel, Kir4.1, and the waterpermeable channel, AQP4, via a dystroglycan-containing complex in astrocytes. Glia 2004;47(02):138-149

47 Tham DKL, Joshi B, Moukhles H. Aquaporin-4 cell-surface expression and turnover are regulated by dystroglycan, dynamin, and the extracellular matrix in astrocytes. PLoS One 2016;11(10):e0165439

48 Nakada T, Kwee IL. Fluid dynamics inside the brain barrier: current concept of interstitial flow, glymphatic flow, and cerebrospinal fluid circulation in the brain. Neuroscientist 2019;25(02):155-166

49 Nakada T, Kwee I, Igarashi H, Suzuki Y. Aquaporin-4 functionality and Virchow-Robin space water dynamics: physiological model for neurovascular coupling and glymphatic flow. Int J Mol Sci 2017;18(08):1798

50 Gram M, Sveinsdottir S, Cinthio M, et al. Extracellular hemoglobin - mediator of inflammation and cell death in the choroid plexus following preterm intraventricular hemorrhage. J Neuroinflammation 2014;11(01):200 
51 McAllister JP, Guerra MM, Ruiz LC, et al. Ventricular zone disruption in human neonates with intraventricular hemorrhage. J Neuropathol Exp Neurol 2017;76(05):358-375

52 Hasan-Olive MM, Enger R, Hansson H-A, Nagelhus EA, Eide PK. Pathological mitochondria in neurons and perivascular astrocytic endfeet of idiopathic normal pressure hydrocephalus patients. Fluids Barriers CNS 2019;16(01):39-55

53 Verkman AS, Tradtrantip L, Smith AJ, Yao X. Aquaporin water channels and hydrocephalus. Pediatr Neurosurg 2017;52(06): 409-416

54 Nakada T. Virchow-Robin space and aquaporin-4: new insights on an old friend. Croat Med J 2014;55:328-336

55 Adzick NS, Thom EA, Spong CY, et al; MOMS Investigators. A randomized trial of prenatal versus postnatal repair of myelomeningocele. N Engl J Med 2011;364(11):993-1004

56 McLone DG, Knepper PA. The cause of Chiari II malformation: a unified theory. Pediatr Neurosci 1989;15(01):1-12

57 Cosmi EV, Anceschi MM, Cosmi E, Piazze JJ, La Torre R. Ultrasonographic patterns of fetal breathing movements in normal pregnancy. Int J Gynaecol Obstet 2003;80(03):285-290

58 Sival DA, Guerra M, den Dunnen WF, et al. Neuroependymal denudation is in progress in full-term human foetal spina bifida aperta. Brain Pathol 2011;21(02):163-179

59 de Wit OA, den Dunnen WF, Sollie KM, et al. Pathogenesis of cerebral malformations in human fetuses with meningomyelocele. Cerebrospinal Fluid Res 2008;5(01):4-13

60 Beck J, Gralla J, Fung C, et al. Spinal cerebrospinal fluid leak as the cause of chronic subdural hematomas in nongeriatric patients. J Neurosurg 2014;121(06):1380-1387

61 Beck J, Raabe A, Schievink WI, et al. Posterior approach and spinal cord release for $360^{\circ}$ repair of dural defects in spontaneous intracranial hypotension. Neurosurgery 2019;84(06):E345-E351

62 Wu C, Guan D, Ren M, et al. Aminophylline for treatment of postdural puncture headache: a randomized clinical trial. Neurology 2018;90(17):e1523-e1529

63 Van Ombergen A, Jillings S, Jeurissen B, et al. Brain ventricular volume changes induced by long-duration spaceflight. Proc Natl Acad Sci U S A 2019;116(21):10531-10536

64 Van Ombergen A, Jillings S, Jeurissen B, et al. Brain tissue-volume changes in cosmonauts. N Engl J Med 2018;379(17):1678-1680

65 Ludwig H-C, Frahm J, Gärtner J, Dreha-Kulaczewski S. Breathing drives CSF: Impact on spaceflight disease and hydrocephalus. Proc Natl Acad Sci U S A 2019;116(41):20263-20264

66 Eide PK, Hansson H-A. Astrogliosis and impaired aquaporin-4 and dystrophin systems in idiopathic normal pressure hydrocephalus. Neuropathol Appl Neurobiol 2018;44(05):474-490

67 Hasan-Olive MM, Hansson H-A, Enger R, Nagelhus EA, Eide PK. Blood-brain barrier dysfunction in idiopathic intracranial hypertension. J Neuropathol Exp Neurol 2019;78(09):808-818

68 Castañeyra-Ruiz L, Hernández-Abad LG, Carmona-Calero EM, Castañeyra-Perdomo A, González-Marrero I. AQP1 overexpression in the CSF of obstructive hydrocephalus and inversion of its polarity in the choroid plexus of a Chiari malformation type ii case. J Neuropathol Exp Neurol 2019;78(07):641-647

69 Gabrion J, Maurel D, Clavel B, et al. Changes in apical organization of choroidal cells in rats adapted to spaceflight or head-down tilt. Brain Res 1996;734(1-2):301-315

70 Masseguin C, Corcoran M, Carcenac C, et al. Altered gravity downregulates aquaporin-1 protein expression in choroid plexus. J Appl Physiol (1985) 2000;88(03):843-850
71 Trillo-Contreras JL, Ramírez-Lorca R, Hiraldo-González L, et al. Combined effects of aquaporin- 4 and hypoxia produce age-related hydrocephalus. Biochim Biophys Acta Mol Basis Dis 2018;1864 (10):3515-3526

72 Wang Y, Tajkhorshid E. Nitric oxide conduction by the brain aquaporin AQP4. Proteins 2010;78(03):661-670

73 Chachlaki K, Prevot V. Nitric oxide signalling in the brain and its control of bodily functions. Br J Pharmacol 2020;177(24): 5437-5458

74 Barbaresi P, Fabri M, Mensà E Characterization of NO-producing neurons in the rat corpus callosum. Brain Behav 2014;4(03): 317-336

75 Li D, Shirakami G, Zhan X, Johns RA. Regulation of ciliary beat frequency by the nitric oxide-cyclic guanosine monophosphate signaling pathway in rat airway epithelial cells. Am J Respir Cell Mol Biol 2000;23(02):175-181

76 Eisenhut M, Choudhury S. In premature newborns intraventricular hemorrhage causes cerebral vasospasm and associated neurodisability via heme-induced inflammasome-mediated interleukin-1 production and nitric oxide depletion. Front Neur 2017;8:423

77 Campos-Ordoñez T, Herranz-Pérez V, Chaichana KL, et al. Longterm hydrocephalus alters the cytoarchitecture of the adult subventricular zone. Exp Neurol 2014;261:236-244

78 Vaziri ND, Ding Y, Sangha DS, Purdy RE. Upregulation of NOS by simulated microgravity, potential cause of orthostatic intolerance. J Appl Physiol (1985) 2000;89(01):338-344

79 Kawada T. Obstructive sleep apnea in patients with idiopathic normal-pressure hydrocephalus. J Neurol Sci 2019;397:155

80 Román GC, Jackson RE, Fung SH, Zhang YJ, Verma AK. Sleepdisordered breathing and idiopathic normal-pressure hydrocephalus: recent pathophysiological advances. Curr Neurol Neurosci Rep 2019;19(07):39-48

81 Hasan-Olive MM, Enger R, Hansson H-A, Nagelhus EA, Eide PK. Loss of perivascular aquaporin-4 in idiopathic normal pressure hydrocephalus. Glia 2019;67(01):91-100

82 Long X, Foussier J, Fonseca P, Haakma R, Aarts RM. Respiration amplitude analysis for REM and NREM sleep classification. Annu Int Conf IEEE Eng Med Biol Soc 2013;2013:5017-5020

83 Rainey-Smith SR, Mazzucchelli GN, Villemagne VL, et al; AIBL Research Group. Genetic variation in Aquaporin-4 moderates the relationship between sleep and brain $A \beta$-amyloid burden. Transl Psychiatry 2018;8(01):47

84 Benveniste H, Lee H, Volkow ND. The glymphatic pathway: waste removal from the CNS via cerebrospinal fluid transport. Neuroscientist 2017;23(05):454-465

85 Marshall-Goebel K, Mulder E, Bershad E, et al. Intracranial and intraocular pressure during various degrees of head-down tilt. Aerosp Med Hum Perform 2017;88(01):10-16

86 Kramer LA, Hasan KM, Sargsyan AE, et al; SPACECOT Investigators Group. Quantitative MRI volumetry, diffusivity, cerebrovascular flow, and cranial hydrodynamics during head-down tilt and hypercapnia: the SPACECOT study. J Appl Physiol (1985) 2017; 122(05):1155-1166

87 Cromwell RL, Scott JM, Downs M, Yarbough PO, Zanello SB, PloutzSnyder L. Overview of the NASA 70-day Bed Rest Study. Med Sci Sports Exerc 2018;50(09):1909-1919

88 Gabrion J, Herbuté S, Oliver J, et al. Choroidal responses in microgravity. (SLS-1, SLS-2 and hindlimb-suspension experiments). Acta Astronaut 1995;36(8-12):439-448 